\title{
Optimal filter approximation by means of a phase-only filter with quantization
}

\author{
Vitaly Kober* \\ Department of Physics, Autonomous University of Barcelona, 08193 Bellaterra, Spain \\ Leonid P. Yaroslavsky* \\ Biomedical Engineering and Instrumentation Program/National Center for Research Resources, \\ National Institutes of Health, Bethesda, Maryland 20892 \\ Juan Campos and Maria J. Yzuel \\ Department of Physics, Autonomous University of Barcelona, 08193 Bellaterra, Spain
}

Received January 5, 1994

\begin{abstract}
Approximate filters based on a phase-only filter for reliable recognition of objects are proposed. Good light efficiency and discrimination capability close to that of the optimal filter can be obtained. Computer simulation results are presented and discussed.
\end{abstract}

Optical correlation methods for pattern recognition have been a subject of intensive investigations since the introduction of the VanderLugt optical correlator. ${ }^{1}$ Different quality criteria and corresponding optimal filters have been suggested to improve pattern recognition. One of the most important quality criterion is the discrimination capability. A linear filter with maximal discrimination capability was proposed by Yaroslavsky and called an optimal filter (OF). ${ }^{2}$ The filter is optimized in terms of the ratio of the peak response to the standard deviation of a background signal to be rejected. The frequency response of the OF is given by

$$
H(\mathbf{f})_{\mathrm{OF}}=X^{*}(\mathbf{f}) /\left\langle|B(\mathbf{f})|^{2}\right\rangle,
$$

where $X^{*}(\mathbf{f})$ is the complex-conjugate spectrum of a target, $B(\mathbf{f})$ is the spectrum of the background signal to be rejected, and \langle\rangle represents a power spectrum estimation operator. The way in which the power spectrum estimation $\left\langle|B(\mathbf{f})|^{2}\right\rangle$ is evaluated depends on the concrete pattern recognition task. ${ }^{3,4}$

A serious drawback in optical implementation of the $\mathrm{OF}$ is its low light efficiency. A filter with maximum light efficiency was proposed by Horner and Gianino and called a phase-only filter (POF). ${ }^{5}$ The amplitude transmission of the filter can be written as

$H(\mathbf{f})_{\mathrm{POF}}=\frac{X^{*}(\mathbf{f})}{|X(\mathbf{f})|}=\left\{\begin{array}{ll}\exp [-i \phi(\mathbf{f})] & |X(\mathbf{f})| \neq 0 \\ 0 & \text { otherwise }\end{array}\right.$,

where $\phi(\mathbf{f})$ is the phase of the target spectrum $X(\mathbf{f})$. Another advantage of the POF is that it can be implemented on available spatial light modulators. One disadvantage of the POF is its poor discrimination capability $^{3}$ for a low-contrast target embedded on a complicated background scene. To improve the performance of the POF, Kumar and Bahri ${ }^{6}$ introduced the concept of the support region. This means that the transmission of the POF is made equal to zero at some frequencies. Methods to calculate support regions to optimize different performance criteria or multicriteria were proposed.?

The approach that we suggest is a different one. We propose to approximate a given filter (in this case the OF) by using, sequentially or in parallel, several filters derived from the same POF by applying different regions of support.

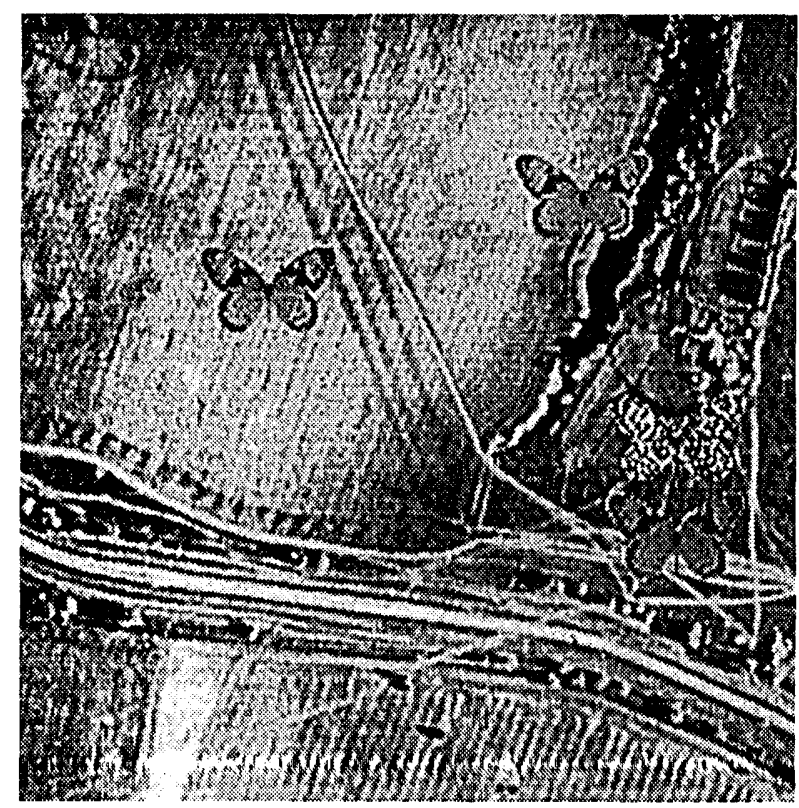

Fig. 1. Aerial photograph with three butterfly targets that was used as the input scene. Scene: mean, 128; standard deviation, 40; $\min , 0 ; \max , 255$. Target: mean, 132; $\min , 90 ; \max , 147$. 
Table 1. Normalized Intensity Correlation Peaks Obtained for the Input Scene in Fig. 1 for the Different Filters

\begin{tabular}{lcllllc}
\hline Filter & $N$ & $T-1$ & $T-2$ & $T-3$ & $F$ & $D$ \\
\hline OF & - & 0.97 & 1 & 0.25 & 0.04 & 0.16 \\
POF & - & 0.005 & 1 & 0.256 & 0.256 & 51.2 \\
AF-POF & 2 & 0.92 & 1 & 0.16 & 0.03 & 0.187 \\
AF-POF & 5 & 0.96 & 1 & 0.23 & 0.036 & 0.162 \\
AF-POF & $256 \mathrm{U}^{a}$ & 0.7 & 1 & 0.23 & 0.12 & 0.52 \\
\hline
\end{tabular}

${ }^{a}$ Denotes the case of uniform quantization.

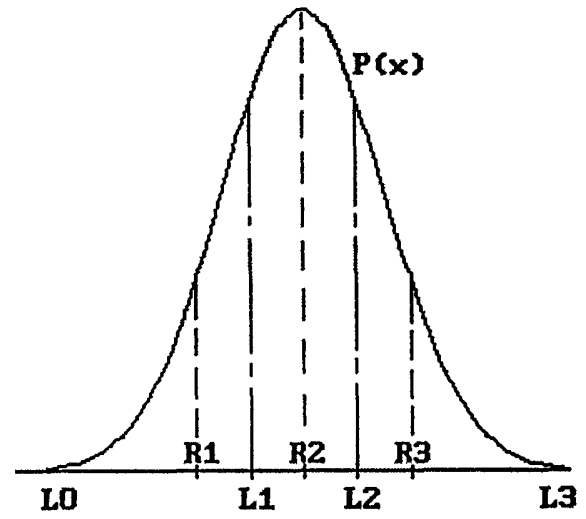

Fig. 2. Illustration of nonuniform optimal quantization.

In our computer simulation the input scene shown in Fig. 1 was used. The scene has $256 \times 256$ samples. Three copies of the target and a false butterfly object are placed on the scene. Rows 1 and 2 of Table 1 show the results obtained with the OF and the POF, respectively. The columns labeled $T-1, T-2$, and $T-3$ give the correlation values corresponding to the three butterfly targets present in the scene at coordinates $(82,92),(182,62),(212,169)$, respectively. The column labeled $F$ corresponds to the false but terfly object $(212,141)$. The column labeled $D$ gives the discrimination capability, defined as the ratio of the maximal cross-correlation peak to the minimum of the three autocorrelation peaks. The table illustrates the superiority of the OF over the POF in terms of discrimination capability.

Now we describe the proposed method to approximate the OF. The relationship between the OF and the POF can be written as follows:

$$
H(\mathbf{f})_{\mathrm{OF}}=\frac{X^{*}(\mathbf{f})}{|X(\mathbf{f})|} \frac{|X(\mathbf{f})|}{\left\langle|B(\mathbf{f})|^{2}\right\rangle}=H(\mathbf{f})_{\mathrm{POF}} F(\mathbf{f})_{\mathrm{POF}}
$$

where $F(\mathbf{f})_{\mathrm{POF}}=|X(\mathbf{f})| /\left\langle\left. B(\mathbf{f})\right|^{2}\right\rangle$ is a correcting factor. In this way we decompose the optimal filter into two filters in cascade: the POF and a correcting filter. A new class of approximate filters (AF's) can be obtained by multiplication of the POF $\left[H(\mathrm{~F})_{\mathrm{POF}}\right]$ by a quantized version of the correcting factor $\left[F(\mathbf{f})_{\mathrm{POF}}\right]$. Let us first describe the quantization process of a signal in general. According to the pointwise quantization concept, the whole dynamic range of a signal (from its minimum to its maximum) is split into a given number of intervals. A representative level is substituted for the signal values within an interval. The representative levels and interval limits depend on the selected criterion for reproducing the signal values by their quantized values. To select the representative levels and interval limits, we use the method known in communication theory as optimal signal quantization. ${ }^{8}$ According to this method, for the case of a minimum squared-error criterion, optimal values of the representative levels and interval limits are given as follows:

$$
\begin{aligned}
R_{i} & =\int_{L_{i-1}}^{L_{i}} x p(x) \mathrm{d} x / \int_{L_{i-1}}^{L_{i}} p(x) \mathrm{d} x, \\
L_{i} & =\left(R_{i}+R_{i+1}\right) / 2,
\end{aligned}
$$

where $R_{i}$ is the representative level of the $i$ th interval, $i=1 \ldots N ; L_{i-1}$ and $L_{i}$ are the limits of the interval; $N$ is the number of intervals; and $p(x)$ is the histogram of the signal values to be quantized. To illustrate the optimal distribution of interval limits and representative levels, we show in Fig. 2 an example of a given signal with a histogram $p(x)$. The number of intervals in Fig. 2 is equal to 3 . We want to emphasize that the interval limits are not distributed uniformly. The histogram of typical correcting factors for real scenes looks like that shown in Fig. 3, calculated for the input scene in Fig. 1. Therefore a nonuniform quantization based on Eq. (4) should be used for a correct approximation of the correcting factor. The quantized factor, which we call $\tilde{F}(\mathbf{f})_{\text {POF }}$, can be represented as a sum, given by

$$
\tilde{F}(\mathbf{f})_{\mathrm{POF}}=\sum_{i=1}^{N} R_{i} B_{i}(\mathbf{f}),
$$

where $R_{i}$ is the corresponding representative value and $B_{i}(\mathbf{f})$ is a binary mask selecting the correctingfactor values for the $i$ th interval:

$$
B_{i}(\mathbf{f})= \begin{cases}1 & L_{i-1}<F(\mathbf{f})_{\mathrm{POF}} \leq L_{i} . \\ 0 & \text { otherwise }\end{cases}
$$

By substituting Eq. (5) into Eq. (3) we obtain an approximation of the OF with a given accuracy by means of the POF and $N$ binary masks:

$$
\begin{aligned}
\tilde{H}(\mathbf{f})_{\mathrm{OF}} & =H(\mathbf{f})_{\mathrm{POF}} \sum_{i=1}^{N} R_{i} B_{i}(\mathbf{f}) \\
& =\sum_{i=1}^{N} R_{i} H(\mathbf{f})_{\mathrm{POF}} B_{i}(\mathbf{f}),
\end{aligned}
$$

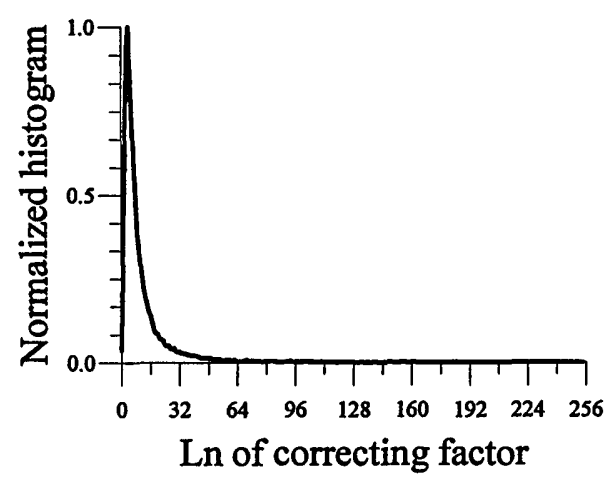

Fig. 3. Histogram of correcting-factor values for the POF calculated for the aerial photograph in Fig. 1. 


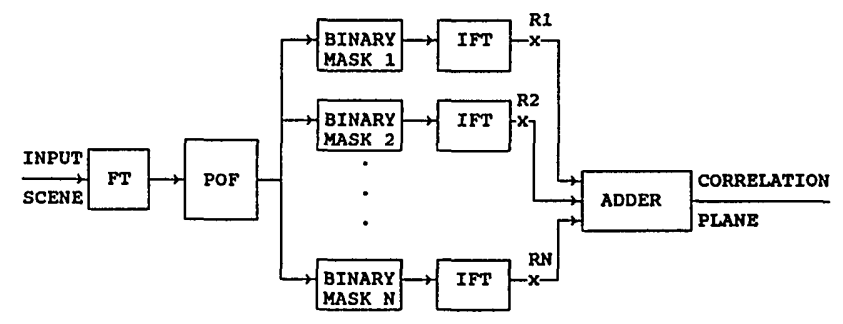

Fig. 4. Block diagram showing the operation performed for pattern recognition by the AF.

where $\tilde{H}(\mathbf{f})_{\mathrm{OF}}$ is the approximate filter AF. The product $H(\mathbf{f})_{\mathrm{POF}} B_{i}(\mathbf{f})$ represents the POF with a region of support $B_{i}(\mathbf{f})$. The correlation with the filter $\tilde{H}(\mathbf{f})_{\mathrm{OF}}$ is the weighted addition of the $N$ correlations obtained with the $N$ filters, $H(\mathbf{f})_{\mathrm{POF}} B_{i}(\mathbf{f})$. The accuracy of the approximation depends on the number of intervals $N$. Table 1 shows the computer simulation results obtained for the input scene in Fig. 1. AF-POF $N$ denotes the AF based on the POF with $N$ binary masks. As the table shows, only a small number of binary masks are necessary for a good approximation of the OF. The light efficiency ${ }^{5}$ obtained with the OF is $5.88 \times 10^{-6}$. In the proposed method we use $N$ filters. Each one will have a different light efficiency. In the case of $N=2$ and $N=5$ the light efficiencies obtained with each filter are $(0.997,0.003)$ and $\left(0.992,5.4 \times 10^{-3}, 1.5 \times 10^{-3}, 0.7 \times 10^{-3}, 10^{-4}\right)$, respectively.

In the last row of Table 1 the case of uniform quantization is presented. Even with 256 uniform quantization intervals, the performance level of the OF is not reached, because more quantization intervals are necessary. So, for the method to be practical, nonuniform quantization intervals have to be used.

Figure 4 shows a block diagram of the method of pattern recognition by means of the proposed AF's. FT and IFT denote forward and inverse Fourier transforms, respectively. $x R i$ denotes multiplication by the $i$ th representative level. For the case in which a small quantity of intervals can approximate the filter, it can be implemented optically. As mentioned above, POF's with different regions of support were already considered as means to improve several performance criteria. 6,7 In our case the masked POF's optimize discrimination capability. An optoelectronic system can be designed to perform the correlation by the proposed method. A spatial light modulator can be employed to introduce into an optical system the binary masks in a time sequence. A computer with a frame grabber may be used to take the correlation snapshots and to accumulate them at a high frame rate. Note that we have approximated the OF, but the procedure may be applied to any filter transmittance. Thus any filter design can be approximated with several filters derived from the same POF by the application of different regions of support.

This study was carried out during V. Kober's stay at the Autonomous University of Barcelona, which was financed by Dirección General de Investigación Científica y Técnica. This research was supported by the Comisión Interministerial de Ciencia y Tecnologia, project TAP93-0667-C03-01. V. Kober and L. P. Yaroslavsky acknowledge a grant for Fundamental Research from the Russian Academy of Sciences.

*Permanent address, Institute of Information Transmission Problems, Russian Academy of Sciences, Yermolovoy Street, 101447 Moscow, Russia.

\section{References}

1. A. B. VanderLugt, IEEE Trans. Inf. Theory IT-10, 139 (1964).

2. L. P. Yaroslavsky, in Advances in Electronics and Electron Physics, P. Hawkes, ed. (Academic, Orlando, Fla., 1986), Vol. 66, pp. 1-140.

3. L. P. Yaroslavsky, Appl. Opt. 31, 1677 (1992).

4. J. Campos, F. Turon, L. P. Yaroslavsky, and M. J. Yzuel, Int. J. Opt. Comput. 2, 341 (1991).

5. J. L. Horner and P. D. Gianino, Appl. Opt. 23, 812 (1984).

6. B. V. K. Vijaya Kumar and Z. Bahri, Appl. Opt. 28, 250 (1989).

7. Ph. Réfrégier, B. V. K. Vijaya Kumar, and C. Hendrix, J. Opt. Soc. Am. A 9, 2188 (1992).

8. J. Max, IRE Trans. Inf. Theory IT-4, 7 (1960). 